\title{
Comparative Renal Protective Effects of Canagliflozin and Telmisartan in a Rat Model of Diabetic Nephropathy
}

Ali F Abdel-Wahab ${ }^{1,2 *}$, Wahid Mahmoud ${ }^{3}$ and Randa Al-Hari$z^{4,5}$

${ }^{1}$ Department of Pharmacology, Cairo University, Giza, Egypt

${ }^{2}$ Department of Pharmacology, Umm al-Qura University, Mecca, Saudi Arabia

${ }^{3}$ Department of Biochemistry, Cairo University, Giza, Egypt

${ }^{4}$ Department of Internal Medicine, Cairo University, Giza, Egypt

${ }^{5}$ Department of Internal Medicine, Ibn Sina National College for Medical Sciences, Jeddah, Saudi Arabia telmisartan, although both drugs produced comparable renoprotective effects. It is concluded that, canagliflozin has a renoprotective effect in diabetic rats similar to that of telmisartan, although through different mechanisms. Thus, SGLT2 inhibitors may represent a promising therapeutic option for the management of type 2 diabetes to slow progression of diabetic nephropathy.

Keywords: Canagliflozin; Diabetic nephropathy; Renoprotection; SGLT2 inhibitors; Telmisartan

\section{Abbreviations}

BUN: Blood Urea Nitrogen

GSH-Px: Glutathione Peroxidase

HbA1c: Glycosylated Hemoglobin

MDA: Malondialdehyde

SOD: Superoxide Dismutase

SGLT2: Sodium Glucose Cotransporter 2

TGF- $\beta 1$ : Transforming Growth Factor Beta1

TNF- $\alpha$ : Tumor Necrosis Factor Alpha

TUNEL: Terminal Transferase-Mediated dUTP Nick-End Labeling

\section{Introduction}

Diabetic nephropathy is a major microvascular complication of diabetes, affecting $20 \%$ to $40 \%$ of diabetic patients [1]. It is an independent risk factor for cardiovascular disease and is associated with increased morbidity and mortality [2]. The glucose-induced oxidative stress and persistent inflammatory condition can cause deleterious renal changes contributing to the pathophysiology of diabetic nephropathy [3]. Increased reactive oxygen species production and reduced availability of antioxidant mechanisms can influence the renal function and structure by modulating cell growth, apoptosis, and inflammatory responses [4]. Also, early diabetic nephropathy is associated with glomerular hyperfiltration, cytokine release and tissue proliferation, processes that have been linked to progression of the kidney disease $[5,6]$.

Inhibitors of the renin-angiotensin system inhibitors are commonly used to decrease glomerular hyperfiltration, reduce proteinuria and delay progression of diabetic nephropathy [7]. Telmisartan, a selective angiotensin receptor blocker have shown to provide renal benefit at all stages of nephropathy in diabetic patients [8,9]. Some investigators have shown that telmisartan also possesses anti-inflammatory and antioxidant properties [10]. However, blockade of the renin-angiotensin system does not completely arrest kidney disease progression and many patients pass to end-stage renal disease [7].

Recently, selective Sodium Glucose Cotransporter 2 (SGLT2) inhibitors have been developed for use in patients with type 2 diabetes [11,12]. Canagliflozin is a selective SGLT2 inhibitor, developed for treatment type 2 diabetes, showing improved glycemic control, both as monotherapy and in combination with other antidiabetics [13,14]. More recently, treatments with SGLT2 inhibitors have been reported to prevent the features of diabetic nephropathy including glomerular hyperfiltration and mesangial matrix expansion in diabetic animal models $[15,16]$. Beyond their antihyperglycemic properties, use of SGLT2 inhibitors was associated with additional non-glycemic benefits including reduction in blood pressure, plasma

Received: April 19, 2016; Accepted: August 11, 2016; Published: August 26 2016 
uric acid and body weight in diabetic patients $[17,18]$. However, the results to date examining the renal protective effects of SGLT2 inhibitors in both clinical studies and animal models of diabetic nephropathy are not consistent. So, the present study examined the possible protective effects of canagliflozin in comparison with telmisartan against kidney injury and dysfunction in diabetic rats. The study also investigated the mechanisms underlying the renoprotective effects, focusing on antioxidant, anti-inflammatory and antiapoptotic properties, in an animal model of diabetic nephropathy.

\section{Materials and Methods}

\section{Animals}

Sixty adult male Wistar rats, weighing 220-250 g (8-10 weeks old), were purchased from the Animal Care Unit, Umm al-Qura University. Animals were housed in cages (four per cage) at constant temperature $\left(22-24^{\circ} \mathrm{C}\right)$ and light-controlled room on an alternating $12: 12-\mathrm{h}$ light/dark cycle. Rats had free access to commercially available rat pellet diet and water ad libitum. The experimental protocol was approved by the local research ethics committee, College of Medicine, Umm al-Qura University. Experimental procedures were carried out in accordance with international guidelines for care and use of laboratory animals.

\section{Drugs and chemicals}

Canagliflozin (Janssen Pharmaceuticals, Inc., UK) was given orally at a dose of 20 or $40 \mathrm{mg} / \mathrm{kg} /$ day [14], for 8 weeks. Telmisartan (Boehringer Ingelheim, Germany) was given orally at a dose of 5 or 10 $\mathrm{mg} / \mathrm{kg} /$ day $[10,19]$, for 8 weeks. All other chemicals and reagents used were of analytical grade obtained from Sigma Chemical Company (St Louis, Mo., USA).

\section{Induction of experimental type 2 diabetes}

After an overnight fasting, diabetes was induced in rats by Intraperitoneal (IP) injection of nicotinamide $(230 \mathrm{mg} / \mathrm{kg}), 15 \mathrm{~min}$ prior to the injection of single dose of streptozotocin $(60 \mathrm{mg} / \mathrm{kg}$, IP), freshly dissolved in $0.1 \mathrm{M}$ cold sodium citrate buffer ( $\mathrm{pH} 4.5$ ) [20]. Control rats received equal volumes of the vehicles alone. The random glucose concentration in blood, taken every other day from tail vein, was determined by means of reactive strips and read with a reflectometer. Rats with moderate hyperglycemia (non-fasting blood glucose above $250 \mathrm{mg} / \mathrm{dl}$ ) were considered diabetic and included in the study.

\section{Treatment protocols}

One week after the overt diabetes, rats were randomly divided into six groups, each of 10 animals. Group 1 served as the non-diabetic control and received only the vehicles. Group 2 served as the diabetic control injected with nicotinamide and streptozotocin as above, but not given any of the test medications. Group 3 and 4 included diabetic rats treated for 8 weeks with oral canagliflozin in a daily dose of 20 and $40 \mathrm{mg} / \mathrm{kg}$, respectively. Group 5 and 6 included diabetic rats treated for 8 weeks with oral telmisartan 5 and $10 \mathrm{mg} / \mathrm{kg} /$ day, respectively. Body weight, water intake and food intake were monitored weekly for all groups. At the end of the study period, all rats are subjected to blood pressure measurements, 24-hour urine collection, and blood and tissue sampling.

\section{Blood pressure measurements}

Blood pressure was measured by the indirect method using a tail cuff blood pressure measuring system (Harvard Apparatus Ltd,
Edenbridge, Kent, England). The mean of three successive measurements was used as the blood pressure estimate. Mean arterial blood pressure was calculated using the equation: diastolic blood pressure $+1 / 3$ (systolic blood pressure- diastolic blood pressure).

\section{Collection of 24-hour urine}

To determine the daily urine volume, each animal was put into the metabolic cage with food and water, awaiting spontaneous micturition. The collected urine for 24 hours was measured and then centrifuged for $5 \mathrm{~min}$ at $1500 \mathrm{rpm}$ and the clear supernatant was stored at $-20^{\circ} \mathrm{C}$ and thawed just before use for determination of creatinine and albumin content. Urinary Albumin Excretion (UAE) rate ( $\mathrm{mg} / 24$ hours) was determined by the Bradford method [21] using a $1 / 20$ dilution of urine in distilled water.

\section{Plasma and tissue sampling}

After an overnight fast, rats were anesthetized with pentobarbital (50 mg/kg IP), and blood samples were obtained by cardiac puncture and collected in tubes containing heparin. The blood samples were then centrifuged for $10 \mathrm{~min}$ at $3000 \mathrm{rpm}$ to obtain clear sera, stored at $-80^{\circ} \mathrm{C}$ until assay. Rats were then sacrificed by exsanguination and the kidneys were quickly removed, washed with ice-cold $0.9 \%$ saline solution, weighed, and processed for tissue biochemical analysis and histopathological examination.

\section{Biochemical measurements}

The separated samples were used for biochemical analysis of plasma glucose, Glycosylated Hemoglobin (HbA1c), UAE, Blood Urea Nitrogen (BUN), serum creatinine, creatinine clearance, TNF- $\alpha$, TGF- $\beta 1$, caspase-3, Total Oxidant Status (TOS) and Total Antioxidant Capacity (TAC) levels.

\section{Blood glucose and glycosylated HbAlc}

Fasting blood glucose levels were determined by glucose meter (Accu-Chek; Roche Diagnostics, Mannheim, Germany), and HbAlc levels were assayed according to the method of Nayak and Pattabiraman [22].

\section{Kidney function parameters}

The BUN concentrations, serum and urinary creatinine levels were measured by an enzymatic assay method using commercial kits (Roche Diagnostics $\mathrm{GmbH}$ ) and Hitachi automatic analyzer (Hitachi Co. Ltd, Tokyo, Japan). Creatinine clearance (reflecting glomerular filtration) was calculated using the formula: Creatinine clearance $(\mathrm{ml} / \mathrm{min})=$ Urine creatinine $\times 24$-h urine volume/serum creatinine $\times 1440$.

\section{Tumor Necrosis Factor alpha (TNF- $\alpha)$}

Serum levels of TNF- $\alpha$ were measured using commercially available ELISA kits according to the manufacturer's instructions (R\&D Systems, Minneapolis, MN, USA). Color intensity of the enzymatic indicator reaction was measured photometrically at $450 \mathrm{~nm}$ in an ELISA plate reader, with a minimum detectable level $0.5 \mathrm{pg} / \mathrm{ml}$.

\section{Transforming Growth Factor beta (TGF- $\beta 1$ )}

Serum levels of TGF- $\beta 1$ were measured using an ELISA kit (Quantikine) according to the manufacturer's guidelines. This assay employs the quantitative sandwich enzyme immunoassay technique and levels are expressed as $\mathrm{ng} / \mathrm{ml}$. 


\section{Serum caspase-3}

Quantitative determination of serum caspase-3 was performed using Colorimetric Assay Kits. It involves conversion of a specific chromogenic substrate for caspase-3 followed by colorimetric detection of colored end-product (p-nitroaniline, p-NA) whose absorbance is directly proportional to the respective caspase- 3 concentration $(\mathrm{U} / \mathrm{ml})$.

\section{Serum total oxidant status and total antioxidant capacity}

The Total Oxidant Status (TOS) in serum was determined using an automated measurement method developed by Erel [23], and results were expressed as micromolar $\mathrm{H}_{2} \mathrm{O}_{2}$ equivalent per litre $\left(\mu \mathrm{mol} \mathrm{H}_{2} \mathrm{O}_{2}\right.$ equiv/L). The Total Antioxidant Capacity (TAC) of serum was determined using the automated measurement method [24], and results were expressed as mmol Trolox equiv/L.

\section{Kidney sample preparation}

Isolated kidneys were used for biochemical analysis of antioxidant status in tissues, TUNEL assay for apoptotic cells, in addition to histopathological examination.

\section{Biochemical studies of tissue oxidative status}

The renal cortex of right kidney was separated, kept frozen in liquid nitrogen at $-80^{\circ} \mathrm{C}$ and subsequently homogenized in cold potassium phosphate buffer $(0.05 \mathrm{~m}, \mathrm{pH}$ 7.4). The homogenates were centrifuged at $5000 \mathrm{rpm}$ for $10 \mathrm{~min}$ at $4^{\circ} \mathrm{C}$ and the supernatant fraction is used for further analyses. Levels of Malondialdehyde (MDA) were determined using colorimetric assay kits according to manufacturer's instructions (Biodiagnostic, Cairo, Egypt) [25]. Activities of Glutathione Peroxidase (GSH-Px) and Superoxide Dismutase (SOD) were assayed using commercial kits (Randox Laboratories, Barcelona, Spain), according to the method of Arthur and Boyne [26].

\section{Terminal Transferase-mediated dUTP Nick-End Labeling} (TUNEL) assay

The apoptotic nuclei were detected by a Transferase-mediated dUTP Nick-End Labeling (TUNEL) assay of kidney sections using peroxidase and counterstaining with hematoxylin and PAS stain [27]. Kidney samples were incubated with an in situ apoptosis detection kit (Takara Bio) according to the manufacturer's protocol. The mean number of TUNEL-positive cells in interstitia per high power field (number/HPF) was determined by observing more than 10 interstitia from each section.

\section{Histopathological examination}

Left kidneys were fixed in $10 \%$ formalin solution, dehydrated and embedded in paraffin. Tissue sections of $5 \mu \mathrm{m}$ thick were taken stained with Hematoxylin and Eosin (H-E). Samples were examined under light microscope by a pathologist unaware of the treatment protocol. The glomerular matrix expansion index was scored in four levels from 0 to 4 , as follows: 0 , normal glomeruli; 1 , matrix expansion occurred in up to $25 \%$ of a glomerulus; 2 , matrix expansion occurred in $25-50 \%$ of a glomerulus; 3 , matrix expansion occurred in $50-75 \%$ of a glomerulus; and 4, matrix expansion occurred in $75-100 \%$ of a glomerulus [28]. Scores were assigned for at least 30 glomeruli from kidney sections for each rat, and the means were calculated.

\section{Statistical analysis}

All data are expressed as mean \pm SEM. Results were analyzed using SPSS computer software package version 19.0 (Chicago, IL, USA).
Multiple groups were compared using one-way Analysis of Variance (ANOVA) followed by post-hoc Kruskal-Wallis and Mann-Whitney U tests. Simple pairwise comparisons were performed using Student's t-test where appropriate. Differences of $\mathrm{P}<0.05$ were considered statistically significant.

\section{Results}

\section{Effect on body weight, blood pressure and hyperglycemia}

Body weight of rats in different groups showed insignificant changes throughout the study period. Measurement of blood pressure showed no significant differences between various groups after 8 weeks of study, apart from a mild but significant reduction in mean arterial blood pressure observed in group 6 , after treatment with high dose of telmisartan (Table 1).

Blood glucose and HbAlc levels were significantly elevated in diabetic groups compared with normal control group. Canagliflozin treatment, at both doses, produced a significant reduction in blood levels of glucose and HbAlc compared with those in the non-treated diabetic group. However, telmisartan treatment did not produce significant effect on blood glucose and HbAlc levels compared with non-treated diabetic rats (Table 1).

\section{Effect on renal function parameters}

Monitoring of kidney function parameters showed significantly higher serum levels of BUN and creatinine in diabetic rats compared with normal control animals. Treatment with canagliflozin, at both doses, produced a significant reduction in BUN and creatinine levels and this reduction was dose-related. Also, telmisartan treatment produced a significant dose-related reduction in BUN and creatinine levels compared with non-treated diabetic rats.

Assessment of albuminuria, a characteristic feature of diabetic nephropathy reflected by albumin excretion rate, showed significant increase in diabetic group compared with non-diabetic control group. Treatment with either canagliflozin or telmisartan leads to significant dose-related reduction in urinary albumin, compared with non-treated diabetic rats.

Daily urine volume showed significant increase in diabetic groups, compared with non-diabetic control rats. Determination of creatinine clearance showed significantly lower levels in non-treated diabetic group and these levels were significantly increased in groups treated with either canagliflozin or telmisartan. No significant differences were observed between effects of high doses of either canagliflozin or telmisartan on BUN, creatinine, creatinine clearance or UAE. Changes in kidney function parameters are summarized in table 2.

\section{Effect on serum levels of inflammatory, apoptotic and oxi- dative markers}

Concentrations of the inflammatory cytokine TNF- $\alpha$ were significantly increased in diabetic group compared with non-diabetic control group $(138.2 \pm 9.8$ versus $64.3 \pm 4.5 \mathrm{pg} / \mathrm{ml})$. These TNF- $\alpha$ levels were significantly reduced by canagliflozin treatment at both doses, and more marked reductions were observed in telmisartan-treated groups especially at a higher dose (Figure 1a).

Similarly, levels of the fibrotic cytokine TGF- $\beta 1$ showed significant increase in diabetic rats compared with non-diabetic controls $(16.4 \pm 1.3$ versus $6.8 \pm 0.4 \mathrm{ng} / \mathrm{ml})$. The elevated TGF- $\beta 1$ levels were 
Citation: Abdel-Wahab AF, Mahmoud W and Al-Harizy R (2016) Comparative Renal Protective Effects of Canagliflozin and Telmisartan in a Rat Model of Diabetic Nephropathy. J Nephrol Renal Ther 2: 010.

- Page 4 of 8 -

\begin{tabular}{|c|c|c|c|c|c|}
\hline$\stackrel{\text { I }}{\text { Non-D }}$ & $\begin{array}{c}\text { II } \\
\text { Diabetic }\end{array}$ & $\begin{array}{c}\text { III } \\
\text { D-C20 }\end{array}$ & $\begin{array}{c}\text { IV } \\
\text { D-C40 }\end{array}$ & $\begin{array}{c}\mathrm{V} \\
\mathrm{D}-\mathrm{T5}\end{array}$ & $\begin{array}{c}\text { VI } \\
\text { D-T10 }\end{array}$ \\
\hline $\begin{array}{l}\text { Body weight (g) } \\
\quad 281 \pm 7.8\end{array}$ & $264 \pm 8.1$ & $261 \pm 8.9$ & $258 \pm 9.4$ & $269 \pm 8.5$ & $272 \pm 7.2$ \\
\hline $\begin{array}{l}\text { Kidney weight }(\mathrm{mg}) \\
\quad 319 \pm 8.6\end{array}$ & $371 \pm 9.8^{*}$ & $356 \pm 9.1$ & $352 \pm 8.2$ & $363 \pm 9.4$ & $348 \pm 8.9$ \\
\hline $\begin{array}{l}\text { Mean blood pressure }(\mathrm{mmHg}) \\
96.3 \pm 4.6\end{array}$ & $104.5 \pm 5.3$ & $98.5 \pm 4.8$ & $95.2 \pm 4.7$ & $91.4 \pm 5.2$ & $83.2 \pm 4.5^{*}+\S$ \\
\hline $\begin{array}{l}\text { Serum glucose }(\mathrm{mg} / \mathrm{dl}) \\
98.9 \pm 7.4\end{array}$ & $257.2 \pm 12.6^{*}$ & $189.6 \pm 9.4 † \S$ & $162.2 \pm 8.3+\S$ & $236.4 \pm 9.1$ & $228.5 \pm 9.7$ \\
\hline $\begin{array}{c}\text { Glycosylated } \mathrm{Hb} \text { A1c (\%) } \\
5.6 \pm 0.2\end{array}$ & $8.7 \pm 0.3^{*}$ & $8.1 \pm 0.2$ & $7.9 \pm 0.2 \dagger$ & $8.5 \pm 0.3$ & $8.4 \pm 0.3$ \\
\hline
\end{tabular}

Table 1: General characteristics of the different rat groups.

Data are presented as mean \pm SEM, $\mathrm{n}=10$. Non-D: Non-Diabetic group, D-C20: Diabetic group treated by Canagliflozin $20 \mathrm{mg} / \mathrm{kg}$, D-C40: Diabetic treated by Canagliflozin $40 \mathrm{mg} / \mathrm{kg}$, D-T5: Diabetic group treated by Telmisartan $5 \mathrm{mg} / \mathrm{kg}, \mathrm{D}-\mathrm{T} 10$ : Diabetic group treated by Telmisartan $10 \mathrm{mg} / \mathrm{kg}$.

*: Significant change vs Control group, $\dagger$ : Significant change vs Non-treated diabetic group, §: Significant change vs Respective dose of the other test drug

\begin{tabular}{|c|c|c|c|c|}
\hline $\begin{array}{c}\text { I } \\
\text { Non-D }\end{array}$ & $\begin{array}{c}\text { II } \\
\text { Diabetic }\end{array}$ & $\begin{array}{c}\text { III } \\
\text { D-C20 }\end{array}$ & $\begin{array}{c}\text { IV } \\
\text { D-C40 }\end{array}$ & $\begin{array}{c}\text { VI } \\
\text { D-T10 }\end{array}$ \\
\hline $\begin{array}{c}\text { Blood urea nitrogen }(\mathrm{mg} / \mathrm{dl}) \\
21.4 \pm 1.3\end{array}$ & $54.6 \pm 3.2^{*}$ & $33.8 \pm 2.0 \dagger$ & $27.6 \pm 1.7 \dagger \ddagger$ & $29.8 \pm 1.8 \dagger$ \\
\hline $\begin{array}{c}\text { Serum creatinine }(\mathrm{mg} / \mathrm{dl}) \\
0.31 \pm 0.02\end{array}$ & $1.16 \pm 0.09^{*}$ & $0.76 \pm 0.05 \dagger$ & $0.51 \pm 0.03 \dagger \ddagger$ & $0.54 \pm 0.03 \dagger \ddagger$ \\
\hline $\begin{array}{c}\text { Albuminuria }(\mathrm{UAE}, \mathrm{mg} / 24 \mathrm{~h}) \\
0.46 \pm 0.04\end{array}$ & $3.7 \pm 0.21^{*}$ & $2.5 \pm 0.14 \dagger$ & $1.8 \pm 0.12 \dagger \ddagger$ & $2.1 \pm 0.12 \dagger$ \\
\hline $\begin{array}{c}\text { Creatinine Clearance }(\mathrm{ml} / \mathrm{min}) \\
9.2 \pm 0.7\end{array}$ & $4.1 \pm 0.5^{*}$ & $7.4 \pm 0.6 \dagger$ & $7.9 \pm 0.6 \dagger$ & $7.8 \pm 0.5 \dagger$ \\
\hline $\begin{array}{c}\text { Urine volume }(\mathrm{ml} / 24 \mathrm{~h}) \\
21.2 \pm 1.0\end{array}$ & $26.5 \pm 1.4^{*}$ & $27.2 \pm 1.3^{*}$ & $28.5 \pm 1.5^{*}$ & $2.11 \dagger \ddagger$ \\
\hline
\end{tabular}

Table 2: Kidney functional parameters in the different rat groups.

Data are presented as mean \pm SEM, $n=10$. Non-D: Non-Diabetic group, D-C20: Diabetic group treated by Canagliflozin 20 mg/kg, D-C40: Diabetic treated by Canagliflozin $40 \mathrm{mg} / \mathrm{kg}$, D-T5: Diabetic group treated by Telmisartan $5 \mathrm{mg} / \mathrm{kg}$, D-T10: Diabetic group treated by Telmisartan $10 \mathrm{mg} / \mathrm{kg}$.

*: Significant change vs Control group, $\uparrow$ : Significant change vs Non-treated diabetic group, $\ddagger$ : Significant change vs Lower dose of same drug

significantly reduced in treated groups given either canagliflozin or telmisartan, with more marked reduction observed in the group treated with high dose of telmisartan, indicating a prominent anti-inflammatory effect (Figure 1b).

Serum levels of the apoptotic marker caspase-3 showed significant elevation in diabetic group compared with non-diabetic control group $(658.7 \pm 26.2$ versus $471.5 \pm 21.6 \mathrm{U} / \mathrm{ml})$. The elevated caspase-3 levels were significantly reduced in treated groups given either canagliflozin or telmisartan, with more marked reduction observed in the group treated with high dose of canagliflozin, indicating a marked anti-apoptotic effect (Figure 1c).

The levels of serum total oxidant status of non-treated diabetic rats were significantly higher compared with control rats $(27.9 \pm 1.8$ versus $16.8 \pm 1.1 \mu \mathrm{M} / \mathrm{L}$ ), and these levels were significantly reduced in treated groups given either canagliflozin or telmisartan, at both dose levels (Figure 1d). In contrast, levels of serum total antioxidant capacity in diabetic rats were significantly lower compared with non-diabetic control rats $(1.8 \pm 0.1$ versus $3.5 \pm 0.3 \mathrm{mM} / \mathrm{L})$. These levels were significantly increased by canagliflozin treatment at the high dose and by telmisartan treatment at both doses (Figure 1e).

\section{Effect on tissue oxidative stress}

Kidney weight and relative kidney weight were significantly higher in diabetic groups compared with control rats, but no significant differences were observed between treated and non-treated

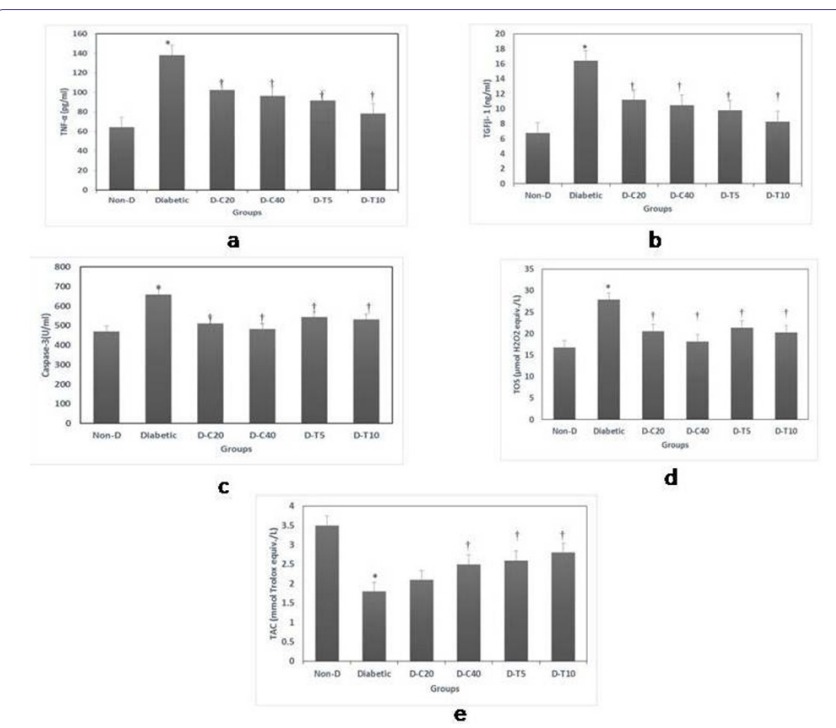

Figure 1: Serum levels of inflammatory, apoptotic and oxidative biomarkers 1a: TNF- $\alpha, 1 \mathrm{~b}$ : TGF- $\beta 1,1 \mathrm{c}$ Caspase-3, $1 \mathrm{~d}$ : TOS (Total Oxidative Status) and 1e: TAC (Total Antioxidant Capacity).

Data are presented as mean \pm SEM, $n=10$. Non-D: Non-Diabetic group, D-C20: Diabetic group treated by Canagliflozin $20 \mathrm{mg} / \mathrm{kg}$, D-C40: Diabetic treated by Canagliflozin $40 \mathrm{mg} / \mathrm{kg}$, D-T5: Diabetic group treated by Telmisartan $5 \mathrm{mg} / \mathrm{kg}$, D-T10: Diabetic group treated by Telmisartan $10 \mathrm{mg} / \mathrm{kg}$. *: Significant change vs Control group, $\dagger$ : Significant change vs Non-treated diabetic group 
diabetic groups (Table 1). This increased kidney weight may reflect renal inflammatory process in diabetic rats.

Tissue MDA levels were significantly increased in kidneys of diabetic rats compared with non-diabetic controls $(7.5 \pm 0.5$ versus $4.6 \pm 0.3 \mathrm{nmol} / \mathrm{mg}$ protein). These levels were significantly reduced by treatment with either canagliflozin or telmisartan (Figure 2a). However, activities of the antioxidant enzymes; GSH-Px, and SOD; showed marked reductions in non-treated diabetic rats, and these levels were significantly increased in groups treated by both canagliflozin and telmisartan (Figure $2 \mathrm{~b} \& 2 \mathrm{c}$ ).

\section{Effect on cellular apoptosis}

Assay of TUNEL staining confirmed that cellular apoptosis was promoted in diabetic kidney compared with normal control group. The number of TUNEL-positive apoptotic cells was significantly decreased in diabetic groups treated with either canagliflozin or telmisartan at both doses, but canagliflozin effect was significantly more marked on renal cell apoptosis than telmisartan (Figure 2d).

\section{Histopathological changes}

Examination of kidney specimens showed several glomerular and tubule-interstitial alterations in diabetic rats compared with non-diabetic control group. Observed lesions included mesangial expansion, nodular sclerosis, hyalinosis, glomerular atrophy, and thickening of basement membrane of both glomeruli and Bowman's capsule. Diabetic rats treated with both canagliflozin and telmisartan presented marked attenuation of the kidney lesions, indicated by the reduction of glomerular fibrosis and diminished glomerulosclerosis. Also, mesangial expansion, hyalinosis and glomerular basement membrane thickening were markedly reduced, compared with non-treated diabetic rats (Figure 3). The glomerular matrix expansion score was found to be highest in the non-treated diabetic group and significantly reduced in a dose-dependent manner in all treated groups, especially those given the higher doses of either canagliflozin or telmisartan (Figure 2e).

\section{Discussion}

The SGLT2 inhibitors are newly developed antidiabetic agents that interfere with renal glucose reabsorption. Many preclinical and clinical studies have demonstrated that SGLT2 inhibitors can reduce blood glucose and $\mathrm{HbAlc}$, independent of pancreatic $\beta$-cell function or insulin sensitivity $[12,13,29]$, but the effects of SGLT2 inhibition on renal structure and function have not been clarified. Therefore, we used a rat model of streptozotocin-nicotinamide induced diabetes to compare the protective effects of canagliflozin and telmisartan, on the development and progression of diabetic nephropathy and to investigate the possible underlying mechanism of action. It has been noted that nicotinamide administration with streptozotocin affords partial $\beta$-cell protection, to provide a model of insulin-deficient, but not insulin-resistant, form of type 2 diabetes characterized by stable, moderate hyperglycemia, with $60 \%$ loss of $\beta$-cell function [20]. This animal model was shown to develop kidney affection and exhibit features of diabetic nephropathy after 8 weeks of hyperglycemia [28].

Current results showed that, after 8 weeks of hyperglycemia, diabetic rats developed significant impairment of renal function and histopathological alterations, associated with increased levels of some inflammatory, oxidant and apoptotic markers. Treatment of diabetic rats with either canagliflozin or telmisartan produced impressive dose-related reduction in the amount of albuminuria in

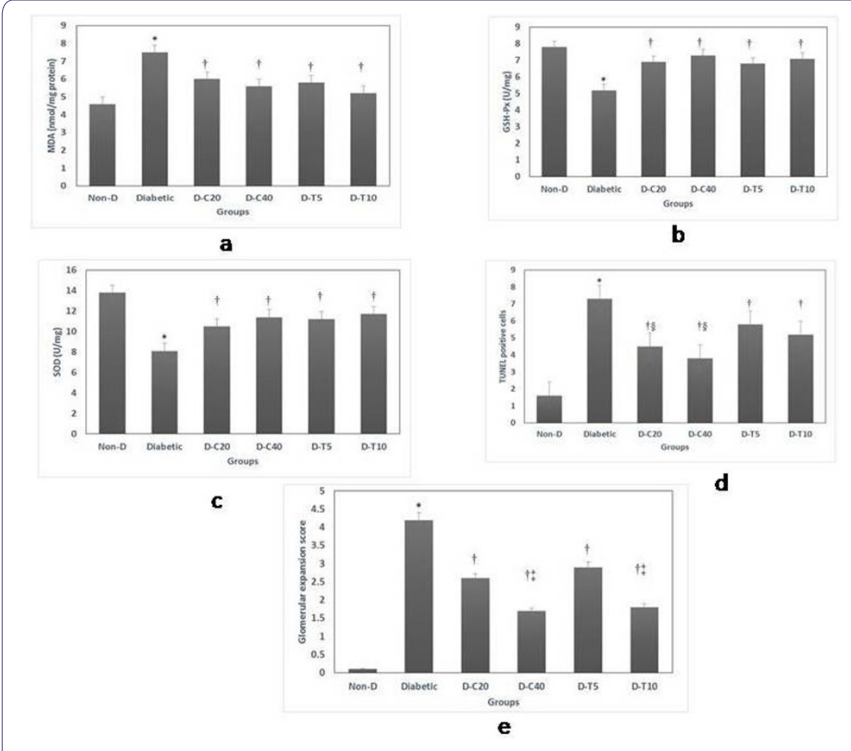

Figure 2: Kidney tissue changes in oxidative status, apoptosis and glomerular matrix expansion score. 2a: MDA (Malondialdehyde), 2b: GSH-Px (Glutathione Peroxidase), 2c: SOD (Superoxide Dismutase), 2d: TUNEL assay and 2e: Glomerular matrix expansion score.

Data are presented as mean \pm SEM, $N=10$. Non-D: Non-Diabetic group, D-C20: Diabetic group treated by Canagliflozin $20 \mathrm{mg} / \mathrm{kg}$, D-C40: Diabetic treated by Canagliflozin $40 \mathrm{mg} / \mathrm{kg}$, D-T5: Diabetic group treated by Telmisartan $5 \mathrm{mg} / \mathrm{kg}$, D-T10: Diabetic group treated by Telmisartan $10 \mathrm{mg} / \mathrm{kg}$. *: Significant change vs Control group, $\uparrow$ : Significant change vs Non-treated diabetic group, ‡: Significant change vs Lower dose of same drug, §: Significant change vs Respective dose of the other test drug

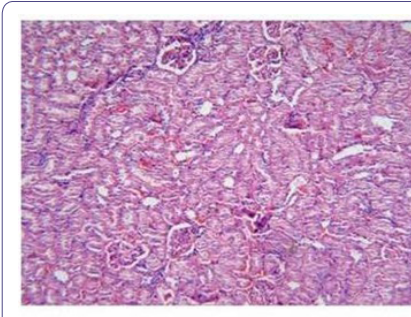

a

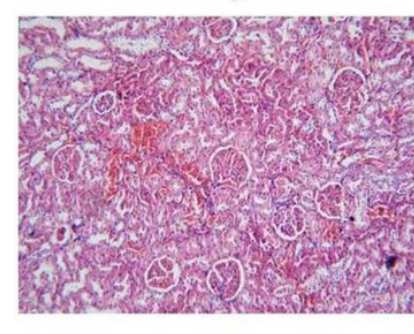

c

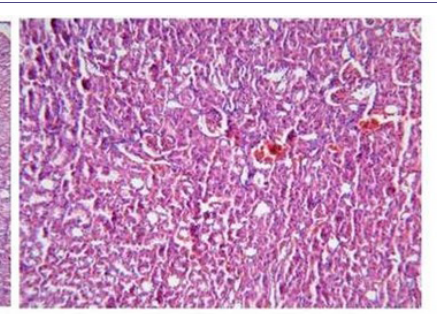

b

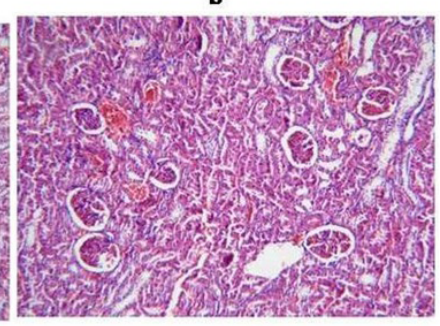

d
Figure 3: Light microscopic findings in kidney specimens isolated from different rat groups (H-E). 3a: Normal control; 3b: Diabetic group, showing mesangial expansion, nodular sclerosis, and thickening of basement membranes; 3c: Diabetic rats treated by canagliflozin $40 \mathrm{mg} / \mathrm{kg}$, showing diminished glomerulosclerosis, and normal basement membrane; $3 \mathrm{~d}$ : Diabetic group treated by telmisartan $10 \mathrm{mg} / \mathrm{kg}$, showing marked attenuation of kidney lesions.

parallel with the decrease in other renal function parameters. Both treatments lead to reduced levels of markers of inflammation, oxidative stress and apoptosis in diabetic rats. Moreover, kidney tissue oxidative stress, renal cell apoptosis and histopathological alterations were attenuated in all treated groups. The renal protective effect of canagliflozin may be related, in part, to amelioration of hyperglycemia 
and decreased HbAlc observed in canagliflozin-treated rats. However, telmisartan treatment produced similar renal protective effects without detectable reduction of blood glucose or HbAlc levels.

Many pathogenetic mechanisms have been suggested to contribute to the development of diabetic nephropathy. It is widely known that prolonged hyperglycemia can induce inflammatory changes and microalbuminuria progressing to nephropathy [30]. In diabetic kidney, macrophage accumulates and its activation is associated with glomerular immune complex deposition, increased cytokine production, and progressive fibrosis [31]. Renal expression of TNF- $\alpha$, was demonstrated to increase in diabetes, contributing to the development of nephropathy [32]. Also, TGF- $\beta 1$, is recognized as an important factor in the pathogenesis of diabetic nephropathy by mediating extracellular matrix accumulation and accelerating glomerular fibrosis in diabetic kidney [33]. Current results showed that treatment with either canagliflozin or telmisartan, produced significant reduction in the elevated levels of TNF- $\alpha$ and TGF- $\beta 1$, and telmisartan was more potent in this respect. Telmisartan activation of the nuclear Peroxisome Proliferator-Activated Receptor- $\gamma$ (PPAR- $\gamma$ ) may contribute to its effect on inflammatory markers and amelioration of kidney disease [34]. These anti-inflammatory effects could alleviate matrix accumulation in the diabetic kidney as observed in histopathological examination.

Moreover, several studies have demonstrated the importance of oxidative stress and apoptosis in the pathogenesis of diabetic nephropathy $[3,35]$. The elevated total oxidant status and decreased antioxidant capacity of serum were corrected by both canagliflozin and telmisartan treatment. Furthermore, the kidney of treated diabetic rats showed lower MDA levels and higher activities of antioxidant enzymes, GH-Px and SOD. Taken together, these findings suggest that canagliflozin and telmisartan treatments can attenuate diabetes-induced oxidative stress in the kidney of diabetic rats. In addition, canagliflozin treatment reduced serum levels of caspase-3 and decreased number of TUNEL-positive apoptotic cells in a dose-dependent manner. Telmisartan treatment produced a similar effect, although to a lesser degree than canagliflozin. Consistent with the above results, treated diabetic rats showed marked morphologic improvement of kidney lesions with attenuated glomerular sclerosis and mesangial matrix expansion, especially at the high doses of either canagliflozin or telmisartan. Therefore, we propose that the observed renoprotective effects in treated diabetic rats could be a consequence of an antioxidant and antiapoptotic effects.

A few studies have addressed the renal protective effects of SGLT2 inhibitors in comparison with the renin-angiotensin system inhibitors. Kojima et al., [16], found that control of hyperglycemia with luseogliflozin, given alone or in combination with lisinopril, could attenuate the development of renal injury in diabetic Dahl salt-sensitive rats treated with streptozotocin. Also, Komala et al., [36], reported that empagliflozin does not have renoprotective benefits independent of glucose lowering, while telmisartan attenuated albuminuria, improved glomerulosclerosis and tubulointerstitial inflammation, in endothelial nitric oxide synthase knockout mice with streptozotocin-induced diabetes. This may indicate that SGLT2 inhibitors have lower renoprotective effect in type 1 diabetes than angiotensin inhibitors. Telmisartan has been shown to improve endothelial function in patients with normoalbuminuria, delays progression to overt nephropathy in patients with microalbuminuria and reduces proteinuria in patients with macroalbuminuria $[8,9]$. Some investigators have shown that telmisartan also possesses anti-inflammatory and antioxidant properties [10,19]. Telmisartan was shown to be more effective than valsartan in lowering albumin excretion and reducing renal inflammation in a rat model of metabolic syndrome [37]. Proposed mechanisms for renal protection include decreased glomerular pressure, reduced albuminuria and suppressed collagen formation by decreasing TGF- $\beta 1$ stimulated by angiotensin [7].

There are some data to support the potential for a direct renoprotective effect arising from inhibition of SGLT2, including actions to attenuate diabetes-associated hyperfiltration and tubular hypertrophy, as well as reduce the tubular toxicity of glucose. The SGLT2 inhibitors can produce a renal protective effect through reduced glomerular hyperfiltration and decreased intraglomerular pressure by activating tubule-glomerular feedback, comparable to the effect of renin-angiotensin inhibitors that act by dilating efferent arterioles. This is supported by data demonstrating that canagliflozin reduces estimated GFR and proteinuria within three weeks in patients with type 2 diabetes and estimated GFR values between $30-50 \mathrm{ml} / \mathrm{min} / 1.73 \mathrm{~m}^{2}$ [13]. Similar reduction in GFR has been observed to occur with empagliflozin treatment in patients with type 2 diabetes and chronic kidney disease [38].

Some studies have demonstrated significant reductions in albumin excretion in experimental models, independent of its effects on blood pressure or glucose control. It has been demonstrated that, tofogliflozin can reduce albuminuria and glomerular hypertrophy in $\mathrm{db} / \mathrm{db}$ mice [39]. Also, luseogliflozin slowed the progression of diabetic nephropathy in diabetic rats [40]. Moreover, dapagliflozin was shown to slow progression of diabetic nephropathy with reduced hyperglycemia and albuminuria in $\mathrm{db} / \mathrm{db}$ mice. Dapagliflozin also suppressed apoptosis and decreased caspase-12 expression in cultured renal proximal tubular cells [41]. Also, Tahara et al., [42], have shown that, ipragliflozin can reduce plasma and liver levels of markers of oxidative stress and inflammation, with improving glycemic control in diabetic mice. On the other hand, Vallon et al., [43], have shown that SGLT2 knockout attenuated hyperglycemia and glomerular hyperfiltration, but not renal injury markers, oxidative stress or inflammation in streptozotocin-induced diabetes model. This discrepancy may be related to the different animal model of diabetes used and the toxic effect of streptozotocin itself. A recent clinical trial suggests that dapagliflozin reduces albuminuria in diabetic patients than placebo, despite having little effect on glycemic control [44]. Current results demonstrated that, the renal protective effect of canagliflozin was comparable and not inferior to that observed with telmisartan in diabetic rats. Canagliflozin showed less anti-inflammatory but more anti-apoptotic effects than telmisartan, and both drugs improved the kidney function and slowed progression of diabetic nephropathy in a rat model of streptozotocin-nicotinamide induced diabetes. However, further studies are needed to assess the possible additive effects of combining both renin-angiotensin inhibitors with SGLT2 inhibitors on renal hemodynamics and kidney protection in type 2 diabetes.

\section{Conclusion}

The SGLT2 inhibitor, canagliflozin, possesses a renoprotective effect comparable to that produced by telmisartam, reducing albuminuria and attenuating the pathologic changes of diabetic nephropathy in rats. Although, glucose-lowering action can contribute to its renoprotective effects, canagliflozin probably exerts other renal protective effects beyond the glycemic control. The possible 
Citation: Abdel-Wahab AF, Mahmoud W and Al-Harizy R (2016) Comparative Renal Protective Effects of Canagliflozin and Telmisartan in a Rat Model of Diabetic Nephropathy. J Nephrol Renal Ther 2: 010.

mechanism for slowing the progression of renal disease may involve reducing oxidative stress, decreasing release of inflammatory and apoptotic mediators, thereby attenuating inflammatory and apoptotic processes in the diabetic kidney. Thus, SGLT2 inhibitors represent a promising therapeutic option for management of type 2 diabetes and useful to prevent or delay progression of diabetic nephropathy.

\section{Acknowledgement}

The authors would like to thank Dr. Mohammad Al-Mesallamany, from the pathology department, Cairo University; for kind assistance in the histopathological evaluation.

\section{Declaration of Conflicting Interest}

The authors declare that there is no conflict of interest.

\section{References}

1. Adler Al, Stevens RJ, Manley SE, Bilous RW, Cull CA, et al. (2003) Development and progression of nephropathy in type 2 diabetes: the United Kingdom Prospective Diabetes Study (UKPDS 64). Kidney Int 63: 225-232.

2. Chronic Kidney Disease Prognosis Consortium, Matsushita K, van der Velde M, Astor BC, Woodward M, et al. (2010) Association of estimated glomerular filtration rate and albuminuria with all-cause and cardiovascular mortality in general population cohorts: a collaborative meta-analysis. Lancet 375: 20732081.

3. Giacco F, Brownlee M (2010) Oxidative stress and diabetic complications Circ Res 107: 1058-1070

4. Navarro-González JF, Mora-Fernández C, Muros de Fuentes M, GarcíaPérez J (2011) Inflammatory molecules and pathways in the pathogenesis of diabetic nephropathy. Nat Rev Nephrol 7: 327-340.

5. Miller JA (1999) Impact of hyperglycemia on the renin angiotensin system in early human type 1 diabetes mellitus. J Am Soc Nephrol 10: 1778-1785.

6. Ziyadeh FN (2004) Mediators of diabetic renal disease: the case for tgf-Beta as the major mediator. J Am Soc Nephrol 15 1: 55-57.

7. Lewis EJ, Hunsicker LG, Clarke WR, Berl T, Pohl MA, et al. (2001) Renoprotective effect of the angiotensin-receptor antagonist irbesartan in patients with nephropathy due to type 2 diabetes. N Engl J Med 345: 851-860.

8. Makino H, Haneda M, Babazono T, Moriya T, Ito S, et al. (2007) Prevention of transition from incipient to overt nephropathy with telmisartan in patients with type 2 diabetes. Diabetes Care 30: 1577-1578.

9. Bakris G, Burgess E, Weir M, Davidai G, Koval S, et al. (2008) Telmisartan is more effective than losartan in reducing proteinuria in patients with diabetic nephropathy. Kidney Int 74: 364-369.

10. Halici Z, Bilen H, Albayrak F, Uyanik A, Cetinkaya R, et al. (2009) Does telmisartan prevent hepatic fibrosis in rats with alloxan-induced diabetes? Eur $\mathrm{J}$ Pharmacol 614: 146-152

11. Kim Y, Babu AR (2012) Clinical potential of sodium-glucose cotransporter 2 inhibitors in the management of type 2 diabetes. Diabetes Metab Syndr Obes 5: 313-327.

12. Bays H (2013) Sodium Glucose Co-transporter Type 2 (SGLT2) Inhibitors: Targeting the Kidney to Improve Glycemic Control in Diabetes Mellitus. Diabetes Ther 4: 195-220.

13. Yale JF, Bakris G, Cariou B, Yue D, David-Neto E, et al. (2013) Efficacy and safety of canagliflozin in subjects with type 2 diabetes and chronic kidney disease. Diabetes Obes Metab 15: 463-473.

14. Rosenstock J, Aggarwal N, Polidori D, Zhao Y, Arbit D, et al. (2012) Dose-ranging effects of canagliflozin, a sodium-glucose cotransporter 2 inhibitor, as add-on to metformin in subjects with type 2 diabetes. Diabetes Care 35: 1232-1238.
15. Vallon V, Gerasimova M, Rose MA, Masuda T, Satriano J, et al. (2014) SGLT2 inhibitor empagliflozin reduces renal growth and albuminuria in proportion to hyperglycemia and prevents glomerular hyperfiltration in diabetic Akita mice. Am J Physiol Renal Physiol 306: 194-204.

16. Kojima N, Williams JM, Slaughter TN, Kato S, Takahashi T, et al. (2015) Renoprotective effects of combined SGLT and ACE inhibitor therapy in diabetic Dahl S rats. Physiol Rep 3.

17. Oliva RV, Bakris GL (2014) Blood pressure effects of sodium-glucose co-transport 2 (SGLT2) inhibitors. below J Am Soc Hypertens 8: 330-339.

18. Chino Y, Samukawa Y, Sakai S, Nakai Y, Yamaguchi Jl, et al. (2014) SGLT-2 inhibitor lowers serum uric acid through alteration of uric acid transport activity in renal tubule by increased glycosuria. Biopharm Drug Dispos 7: 10-19.

19. Faruk EM, Salem MY, El-eraky El-azab N (2015) The effect of telmisartan versus garlic on renal cortex of experimentally induced hypertension in rats: a biochemical, histological and immunohistochemical study. J Cell Tissue Res 15: $4727-4736$.

20. Masiello P, Broca C, Gross R, Roye M, Manteghetti M, et al. (1998) Experimental NIDDM: development of a new model in adult rats administered streptozotocin and nicotinamide. Diabetes 47: 224-229.

21. Bradford MM (1976) A rapid and sensitive method for the quantitation of microgram quantities of protein utilizing the principle of protein-dye binding. Anal Biochem 72: 248-254.

22. Nayak SS, Pattabiraman TN (1981) A new colorimetric method for the estimation of glycosylated hemoglobin. Clin Chim Acta 109: 267-274.

23. Erel O (2005) A new automated colorimetric method for measuring total oxidant status. Clin Biochem 38: 1103-1111.

24. Erel O (2004) A novel automated method to measure total antioxidant response against potent free radical reactions. Clin Biochem 37: 112-119.

25. Jentzsch AM, Bachmann H, Fürst $P$, Biesalski HK (1996) Improved analysis of malondialdehyde in human body fluids. Free Radic Biol Med 20: 251-256.

26. Arthur JR, Boyne R (1985) Superoxide dismutase and glutathione peroxidase activities in neutrophils from selenium deficient and copper deficient cattle. Life Sci 36: 1569-1575.

27. Gavrieli Y, Sherman Y, Ben-Sasson SA (1992) Identification of programmed cell death in situ via specific labeling of nuclear DNA fragmentation. J Cell Biol 119: 493-501.

28. Tzeng T, Liou S, Chang CJ, Liu I (2013) Zerumbone, a tropical ginger sesquiterpene, ameliorates streptozotocin-induced diabetic nephropathy in rats by reducing the hyperglycemia-induced inflammatory response. Nut Metabol 10: 64 .

29. Macdonald FR, Peel JE, Jones HB, Mayers RM, Westgate L, et al. (2010) The novel sodium glucose transporter 2 inhibitor dapagliflozin sustains pancreatic function and preserves islet morphology in obese, diabetic rats. Diabetes Obes Metab 12: 1004-1012.

30. Lim AK, Tesch GH (2012) Inflammation in diabetic nephropathy. Mediators Inflamm.

31. Chow FY, Nikolic-Paterson DJ, Atkins RC, Tesch GH (2004) Macrophages in streptozotocin-induced diabetic nephropathy: potential role in renal fibrosis. Nephrol Dial Transplant 19: 2987-2996.

32. Navarro-González JF, Mora-Fernández C (2008) The role of inflammatory cytokines in diabetic nephropathy. J Am Soc Nephrol 19: 433-442.

33. Sharma K, Jin Y, Guo J, Ziyadeh FN (1996) Neutralization of TGF-beta by anti-TGF-beta antibody attenuates kidney hypertrophy and the enhanced extracellular matrix gene expression in STZ-induced diabetic mice. Diabetes 45: $522-530$

34. Baylis C, Atzpodien EA, Freshour G, Engels K (2003) Peroxisome proliferator-activated receptor (gamma) agonist provides superior renal protection versus angiotensin-converting enzyme inhibition in a rat model of type 2 diabetes with obesity. J Pharmacol Exp Ther 307: 854-860. 
Citation: Abdel-Wahab AF, Mahmoud W and Al-Harizy R (2016) Comparative Renal Protective Effects of Canagliflozin and Telmisartan in a Rat Model of Diabetic Nephropathy. J Nephrol Renal Ther 2: 010.

35. Cachofeiro V, Goicochea M, de Vinuesa SG, Oubiña P, Lahera V, et al. (2008) Oxidative stress and inflammation, a link between chronic kidney disease and cardiovascular disease. Kidney Int: 4-9.

36. Komala MG, Gross S, Mudaliar H, Huang C, Pegg K, et al. (2014) Inhibition of Kidney Proximal Tubular Glucose Reabsorption Does Not Prevent against Diabetic Nephropathy in Type 1 Diabetic eNOS Knockout Mice. PLoS ONE 9: 108994.

37. Khan AH, Imig JD (2011) Telmisartan provides better renal protection than valsartan in a rat model of metabolic syndrome. Am J Hypertens 24: 816-821.

38. Barnett AH, Mithal A, Manassie J, Jones R, Rattunde H, et al. (2014) Efficacy and safety of empagliflozin added to existing antidiabetes treatment in patients with type 2 diabetes and chronic kidney disease: a randomised, double-blind, placebo-controlled trial. Lancet Diabetes Endocrinol. 2: 369-384

39. Nagata T, Fukuzawa T, Takeda M, Fukazawa M, Mori T, et al. (2013) Tofogliflozin, a novel sodium-glucose co-transporter 2 inhibitor, improves renal and pancreatic function in db/db mice. Br J Pharmacol 170: 519-531.

40. Kojima N, Williams JM, Takahashi T, Miyata N, Roman RJ (2013) Effects of a new SGLT2 inhibitor, luseogliflozin, on diabetic nephropathy in T2DN rats. J Pharmacol Exp Ther 345: 464-472.
41. Terami N, Ogawa D, Tachibana H, Hatanaka T, Wada J, Nakatsuka A, et al. (2014) Long-Term Treatment with the Sodium Glucose Cotransporter 2 Inhibitor, Dapagliflozin, Ameliorates Glucose Homeostasis and Diabetic Nephropathy in db/db Mice. PLoS ONE 9: 100777.

42. Tahara A, Kurosaki E, Yokono M, Yamajuku D, Kihara R, et al. (2013) Effects of SGLT2 selective inhibitor ipragliflozin on hyperglycemia, hyperlipidemia, hepatic steatosis, oxidative stress, inflammation, and obesity in type 2 diabetic mice. Eur J Pharmacol 715: 246-255.

43. Vallon V, Rose M, Gerasimova M, Satriano J, Platt KA, et al. (2013) Knockout of Na-glucose transporter SGLT2 attenuates hyperglycemia and glomerular hyperfiltration but not kidney growth or injury in diabetes mellitus. Am J Physiol Renal Physiol 304: 156-167.

44. Kohan DE, Fioretto P, Tang W, List JF (2014) Long-term study of patients with type 2 diabetes and moderate renal impairment shows that dapagliflozin reduces weight and blood pressure but does not improve glycemic control. Kidney Int 85: 962-971. 